\title{
Acquisition and extinction of conditioned nicotine analgesic tolerance
}

\author{
JULIAN L. AZORLOSA, CAROLYN E. JOHNSON, and JAMES J. MCCONNELL \\ West Chester University, West Chester, Pennsylvania
}

\begin{abstract}
These studies demonstrated the acquisition and extinction of conditioned tolerance to the analgesic effect of nicotine in rats. In Experiment 1, distinctive environmental cues were either paired or unpaired with nicotine. Following acquisition, the paired group was more tolerant to nicotine than the unpaired and saline groups. Conditioned tolerance was extinguished in the paired group after placebo sessions in the distinctive environment. Experiment 2 examined whether the distinctive environment functioned as a CS or as an occasion setter for injection cues. After acquisition, exposure to the distinctive environment, with or without placebo injections, resulted in extinction. This demonstrates that the distinctive environment served as a CS, not as an occasion setter for injection cues.
\end{abstract}

Repeated administration of many psychoactive drugs results in diminished effectiveness of the drug, a phenomenon named tolerance. A great deal of research has shown that associative learning contributes to tolerance (see Siegel \& Ramos, 2002, for a recent review). Siegel (1975) proposed a model of tolerance in which drug administration was seen as an example of Pavlovian or classical conditioning. Environmental or contextual cues present when the drug is taken are conditioned stimuli (CSs), and the drug is the unconditioned stimulus (US). Compensatory conditioned responses (CRs) develop to counteract the effects of the drug, which are the unconditioned responses (URs). These compensatory CRs are opposite to the drug effects and help maintain homeostasis. More recent versions of this theory have maintained that the URs are not the drug effects but rather the compensatory responses (Ramsay \& Woods, 1997; Siegel \& Allan, 1998). In either case, environmental CSs elicit compensatory CRs, which mediate tolerance. Poulos and Cappell (1991) also proposed that compensatory responses to drugs occur to preserve homeostasis but emphasized behavioral contingencies rather than classical conditioning. Baker and Tiffany (1985) proposed a classical conditioning model in which habituation, not compensatory CRs, mediates tolerance. Paletta and Wagner (1986) suggested that, depending on the particular drug effect, either compensatory responding or habituation is the mechanism of classically conditioned tolerance. Although these theories differ somewhat as to the underlying process, all propose that environmental cues mediate tolerance.

We dedicate this article to the memory of Jim McConnell. This research was funded by a College of Arts and Sciences Support and Development Award to the first author. Correspondence concerning this article should be addressed to J. L. Azorlosa, Department of Psychology, Peoples Building, West Chester University of Pennsylvania, West Chester, PA 19383 (e-mail: jazorlosa@wcupa.edu).
Contextual control of tolerance has been demonstrated with many drugs, including morphine (Siegel, 1975, 1977), ethanol (Brooks, Karamanlian, \& Foster, 2001; Mansfield \& Cunningham, 1980; Siegel, 1987), and some benzodiazepines (Greeley \& Cappell, 1985; Griffiths \& Goudie, 1986; King, Bouton, \& Musty, 1987). In addition, contextual control of tolerance to many different effects of these drugs has been demonstrated (see Siegel, Baptista, Kim, McDonald, \& Weise-Kelly, 2000, for a review). Numerous studies have demonstrated conditioned tolerance to the effects of morphine, including analgesia (Siegel, 1975), hyperthermia (Siegel, 1978), and sedation (Fanselow \& German, 1982). Conditioned tolerance has been shown to the hypothermic (Mansfield \& Cunningham, 1980) and ataxic (Brooks et al., 2001) effects of ethanol as well as the sedative (King et al., 1987) and hypothermic (Greeley \& Cappell, 1985) effects of benzodiazepines.

Many studies have also determined that conditioned tolerance conforms to the principles of classical conditioning found in traditional preparations. Contextual drug tolerance is subject to latent inhibition (Siegel, 1977), conditioned inhibition (Siegel, Hinson, \& Krank, 1981), overshadowing (Walter \& Riccio, 1983), sensory preconditioning, and blocking (Dafters, Hetherington, \& McCartney, 1983). In addition, contextual tolerance can also be extinguished if the contextual cues are presented without the drug. Numerous studies have shown extinction of contextual tolerance to various effects of several drugs, including the analgesic effect of morphine (MacRae \& Siegel, 1987; Millin \& Riccio, 2002; Siegel, 1975; Siegel, Sherman, \& Mitchell, 1980), the hyperthermic effect of morphine (Siegel, 1978) and of ethanol (Mansfield \& Cunningham, 1980), the ataxic effect of ethanol (Brooks et al., 2001; Siegel, 1987), and the sedative effect of midazolam (King et al., 1987) and of morphine (Fanselow \& German, 1982).

Contextual tolerance has also been demonstrated with nicotine. Caggiula and his colleagues have shown that 
environmental cues mediate tolerance to its anorectic effects (Caggiula et al., 1991; Caggiula, Epstein, \& Stiller, 1989), its tachycardiac effect (Epstein, Caggiula, Perkins, McKenzie, \& Smith, 1991), its cortisone-elevating effect (Caggiula et al., 1991; but see Davis, Cepeda-Benito, Harraid, \& Wellman, 2005), and its analgesic effect (Caggiula et al., 1993; Epstein, Caggiula, \& Stiller, 1989). Cepeda-Benito and colleagues have also found contextual control of the analgesic effect of nicotine (CepedaBenito, Davis, Reynoso, \& Harraid, 2005; Cepeda-Benito, Reynoso, \& Erath, 2000; Cepeda-Benito, Reynoso, \& McDaniel, 1998).

In addition to the studies that have examined classically conditioned tolerance to nicotine, several other studies have demonstrated the potential of environmental stimuli to become conditioned to the effects of nicotine. For example, environmental cues paired with nicotine can elicit sensitization of nicotine's increase of locomotor activity (Bevins, Besheer, \& Pickett, 2001; Bevins \& Palmatier, 2003). Caggiula and colleagues have shown that environmental stimuli can serve as secondary reinforcers and promote nicotine self-administration in rats (Caggiula et al., 2001; Caggiula, Donny, White, et al., 2002) and smoking in humans (Caggiula, Donny, Chaudhri, et al., 2002; Caggiula et al., 2001).

However, in comparison with the research on other drugs such as morphine and ethanol, relatively little research has been devoted to classically conditioned tolerance to nicotine. This is surprising, given that nicotine is one of the most widely used psychoactive drugs and over a quarter of American adults are smokers (U.S. Department of Health \& Human Services, 1997). A more complete understanding of conditioned nicotine tolerance would contribute to the development of effective therapies for smoking cessation. As far as we have been able to determine, no published studies have examined whether contextual nicotine tolerance can be extinguished. Because smoking cessation inevitably involves extinction, it is important to demonstrate that a conditioned effect of nicotine can be extinguished. Therefore, the purpose of Experiment 1 was to demonstrate contextual tolerance to the analgesic effect of nicotine and then determine whether this tolerance could be extinguished by presenting the contextual cues without the drug.

\section{EXPERIMENT 1}

\section{Method}

Subjects. Twenty-four male Sprague-Dawley rats were used in this study. They weighed 240-270 g at the start of the experiment. They were housed, in groups of four, in wire mesh cages. The colony room was illuminated from 7 a.m. to 9 p.m. Food and water were available ad lib.

Apparatus, analgesia assessment, and drugs. During a conditioning session, a rat was taken from the home cage (HC), weighed, and taken to its assigned box in a different room, called the distinctive environment (DE), that had distinctive contextual cues that made it quite different from the $\mathrm{HC}$. The sides of the boxes were made of glass ( $25.4 \mathrm{~cm}$ high, $25.4 \mathrm{~cm}$ wide, and $50.8 \mathrm{~cm}$ long), with alternating black and white stripes $(2.54 \mathrm{~cm}$ wide $)$. The floors of the boxes were also glass, and the top was a wire mesh lid. Each box was illuminated by a $25-\mathrm{W}$ red bulb. These bulbs were the only source of illumination in the DE. Extraneous noise was masked by a white noise generator $(75 \mathrm{~dB}$, scale $\mathrm{C})$. Immediately before the start of a session, the boxes were cleaned with a lemon-scented disinfectant. There were eight boxes in the DE. They rested on tables and were spaced $15-20 \mathrm{~cm}$ apart.

Analgesia was assessed with the tail-flick method. During a test, each rat was removed from the cage and gently restrained by hand, with its tail draped over the edge of the table. The tail was immersed up to a black mark on the tail, $2.5 \mathrm{~cm}$ from the tip, in a cup of water taken from a $500-\mathrm{ml}$ jar heated to $52^{\circ} \mathrm{C}( \pm 0.5)$ on a hot plate. A new cup of water was used for each rat. A thermometer in the jar provided constant monitoring of the temperature. The time that it took the rat to flick the tail out of the water was measured with a stopwatch. All analgesia tests were conducted by the same person, who was blind to each rat's group assignment. Pilot work showed that these procedures produced latencies of 2-4 sec in nondrugged rats of the same age.

Injections were given subcutaneously to the dorsal neck area. (-)Nicotine hydrogen tartrate (Sigma, St. Louis, MO) was administered in a $1-\mathrm{ml} / \mathrm{kg}$ vehicle of $.9 \% \mathrm{NaCl}$. The $\mathrm{pH}$ was brought to 7.0 with a dilute $\mathrm{NaOH}$ solution. The dose of nicotine $(2.85 \mathrm{mg} / \mathrm{kg}$, expressed as the salt, $1.0 \mathrm{mg}$ as nicotine base) was chosen on the basis of previous studies that demonstrated conditioned tolerance to the analgesic effect of nicotine (Cepeda-Benito et al., 1998, Experiment 2; Epstein et al., 1989).

Procedure. Two weeks prior to the experiment, the rats were habituated to handling and injections. They received a total of three saline injections. The rats were then randomly assigned to one of three groups $(n=8)$ and given eight conditioning sessions in the DE. During each session, the rats were taken, in groups of 8 , from the $\mathrm{HC}$ to the DE, given an injection, and then immediately placed in the assigned box. Box assignment was counterbalanced between groups. Five and $10 \mathrm{~min}$ after the injection, each rat received mock tail-flick tests. The rat was gently restrained with its tail draped over the edge of the table for $10 \mathrm{sec}$. After $30 \mathrm{~min}$, the rats were returned to the HC. Approximately $24 \mathrm{~h}$ later, $\mathrm{HC}$ injections were given.

The DE sessions were spaced 48 and $72 \mathrm{~h}$ apart; they were run on Mondays, Wednesdays, and Fridays. Rats in the paired group received nicotine in the DE and saline in the HC. The unpaired group received saline in the DE and nicotine in the HC. The saline group received saline in both. Forty-eight hours following the last conditioning session, the test session was administered. This session was identical to the training session, except that all rats received nicotine in the DE. The tail-flick test was conducted 5 and 10 min after injection and placement in the cage. The latency to flick the tail out of the water was recorded. If a rat failed to flick its tail within $20 \mathrm{sec}$, the test was terminated to prevent tissue damage and a score of $20 \mathrm{sec}$ was recorded. Forty-eight hours after the test, a reminder session was run that was identical to a conditioning session.

Following the reminder session, the extinction phase began. This phase was identical to the conditioning sessions, except that all rats received saline injections and there were no $\mathrm{HC}$ injections. There were four extinction sessions. After the last extinction session, all groups were given a second tolerance test in the DE. During training and testing, the experimenters were blind to the rats' group assignments.

\section{Results and Discussion}

There were no appreciable differences between the 5and 10-min test latencies, so these were averaged. Median tail-flick latencies from the analgesic test after acquisition are shown in Figure 1. Groups with prior exposure to nicotine (paired and unpaired) had shorter latencies than did the saline group, which suggests that the prior exposure to the drug produced tolerance. The paired group 


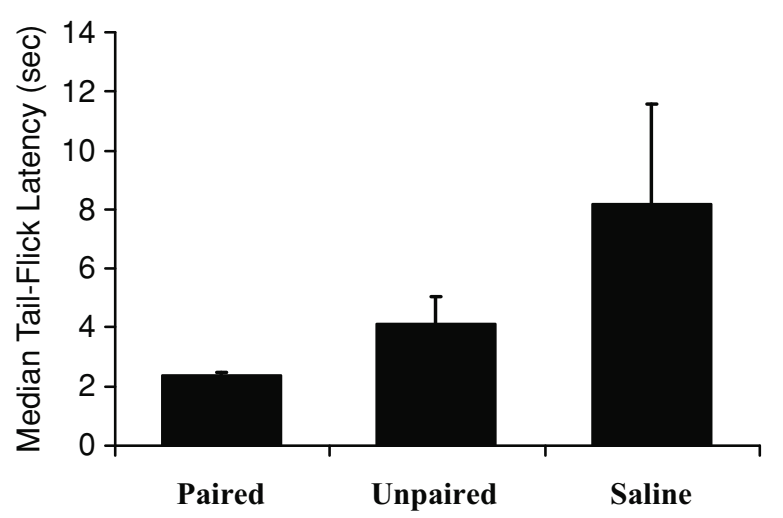

Figure 1. Median tail-flick latency in the postacquisition test in Experiment 1. Error bars represent the semi-interquartile range.

had a shorter mean latency than did the unpaired group, which suggests contextual tolerance. Due to marked heterogeneity of variance $[F(2,21)=13.06, p<.001]$, the latency scores were examined with nonparametric tests. A Kruskal-Wallace test indicated significant differences among groups $\left(\chi^{2}=3.03, p<.01\right)$. Mann-Whitney $U$ tests confirmed the differences suggested in Figure 1. Both the paired and the unpaired groups had significantly shorter latencies than did the saline group $(U=1, p<$ $.01 ; U=13, p<.05$, respectively), and the paired group had shorter latencies than did the unpaired group, $(U=$ $11, p<.05)$.

The results of the test conducted after four extinction trials are shown in Figure 2. Although both the paired and the unpaired groups still had shorter latencies than did the saline group, they are similar to each other. A KruskalWallace test indicated a difference among the groups $\left(\chi^{2}=6.65, p<.05\right)$. Mann-Whitney $U$ tests showed that both the paired and the unpaired groups had significantly shorter scores than did the saline group (both $U_{\mathrm{s}}=5, p<$ $.05)$, but that the paired and unpaired groups did not differ $(U=29, p=.798)$.

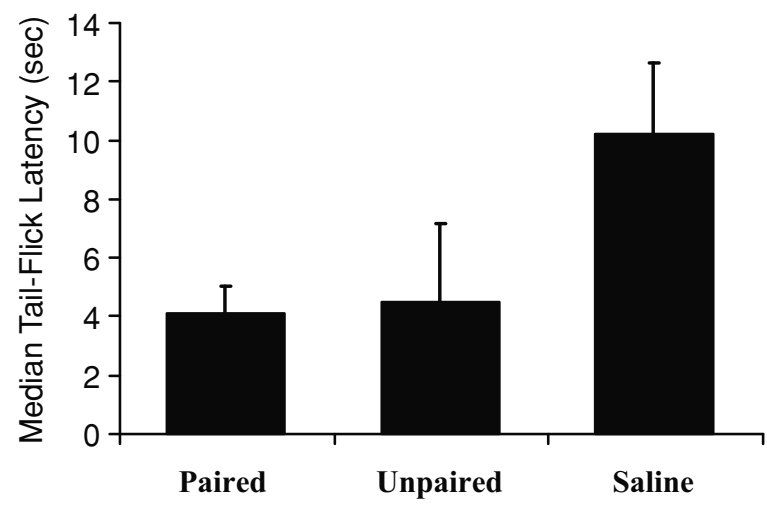

Figure 2. Median tail-flick latency in the postextinction test in Experiment 1. Error bars represent the semi-interquartile range.
The tail-flick tests conducted after the acquisition phase (Figure 1) showed that the paired group had significantly shorter latencies than did the unpaired group. This is evidence of contextual control of tolerance to the analgesic effect of nicotine, which is consistent with previous research. The results of the second tolerance test (Figure 2), in which the paired and unpaired scores were the same, show that conditioned tolerance to the analgesic effect of nicotine can be extinguished if the conditioned stimuli originally paired with nicotine are presented several times without it. Numerous studies have shown that conditioned drug tolerance can be extinguished (see Siegel et al., 2000). The present study is the first demonstration of extinction of conditioned tolerance to any effect of nicotine. These results are consistent with the conditioning model proposed by Siegel (1975; see also Siegel et al., 2000). However, the present study did not address the mechanism of this tolerance, only that it was mediated by contextual cues.

Inspection of Figure 2 also indicates that although the paired and unpaired groups show the same degree of analgesia, their latencies are both substantially shorter than those for the saline group. The shorter latencies of the paired group may reflect incomplete extinction. That the unpaired group still showed tolerance after 10 nicotinefree days may have been due to either dispositional or functional tolerance. Dispositional tolerance results from the increased metabolism or distribution of the drug, which renders a given dose less effective. Functional tolerance results from neuronal changes that make the dose less potent. Tolerance in the unpaired group is unlikely to have been mediated by injection cues as was found by Cepeda-Benito and Tiffany (1995). They found tolerance retention only in unpaired rats that received no saline injections during the retention interval. Our rats showed retention of tolerance even though they received saline injections during the retention phase. A possible reason for this difference in findings is that we gave the injections s.c., whereas they gave the injections i.p., which may have made the injections more salient (Cepeda-Benito \& Tiffany, 1995).

However, the tolerance in the unpaired group could have been due to the retention of another type of conditioned tolerance. Several investigators (see Siegel \& Ramos, 2002, for a review) have found that early drug-onset cues may serve as a CS for later, larger drug effects and come to elicit compensatory responses that mitigate those later effects. Drug onset cues are especially potent with the relatively slow onset of an s.c. injection as opposed to an i.v. injection (Grisel, Wiertelak, Watkins, \& Maier, 1994). In the present study, this analysis would suggest that the early effects of nicotine in the unpaired group served to lessen the later effects, producing a form of conditioned tolerance that was retained during the 10 days from the last $\mathrm{HC}$ injection and the second test. Kim, Siegel, and Patenall (1999) and Walter and Riccio (1983) have suggested that drug-onset cues might potentially compete with or overshadow environmental cues. The unpaired group, in contrast to the paired group, had no contextual cues (like the 
DE) consistently paired with the drug and nothing else, making it possible that the home cage cues were overshadowed by the drug-onset cues. However, the present procedures offer no way to confirm these speculations.

Although we were able to successfully extinguish conditioned tolerance to nicotine, it is not entirely clear what stimulus mediated the tolerance. In the paired/unpaired/ saline design used in the present study and numerous others, the context (the DE) is viewed as the CS. In this view, extinction occurred in the paired group because the rats received exposure to the DE without the drug. However, the paired/unpaired/saline experimental design may result in the formation of a somewhat different associative structure in which the DE serves not as a CS, but as an occasion setter for the injection procedure, which is the actual CS. This is a hierarchical form of classical conditioning in which one stimulus "sets the occasion" for the second stimulus, the CS, to be followed by the US (Holland, 1983). On trials when the occasion-setting stimulus is not presented, the CS is presented but is not followed by the US. The phenomenon of occasion setting also has been called facilitation (Rescorla, 1985) and modulation (Swartzentruber, 1995). In the present study, the DE might potentially have set the occasion for the injection to be followed by nicotine in the paired group. When the paired group received home cage injections of saline, the injection CS was not followed by nicotine.

If the DE served as an occasion setter in Experiment 1, then extinction occurred because the CS (the injection cues) was followed by no US (nicotine) in the presence of the occasion-setting DE. There is ample evidence that contextual cues can serve as occasion setters (see, e.g., Bouton \& Swartzentruber, 1986; Grahame, Hallam, Geier, \& Miller, 1990; Swartzentruber, 1991), and there is evidence for occasion setting in drug tolerance. Ramos, Siegel, and Bueno (2002) found that a flashing light could set the occasion for an injection CS to control tolerance to the hypothermic effect of ethanol.

\section{EXPERIMENT 2}

The purpose of Experiment 2 was to determine the relative roles of the DE and injection cues in the paired/ unpaired design typically used in studies of classically conditioned tolerance. One possibility was that the DE is the CS and the injection cues, because of pretraining and home cage injections, do not enter into an association with the drug. A second possibility was that although the DE is the primary $\mathrm{CS}$, the injection cues also become associated with the drug. Finally, the DE might serve as an occasion setter and the injection cues serve as the CS.

Four groups of rats were given acquisition sessions with nicotine paired with the DE. A fifth group (unpaired) received morphine in the HC. Following acquisition, the extinction phase began. Two of the paired groups did not receive extinction training and remained in the $\mathrm{HC}$. The other two paired groups had extinction sessions in the DE. In the two groups undergoing extinction training, one received a saline injection prior to placement in the assigned cage in the DE and the other did not. If the DE served as an occasion setter for the injection CS, then extinction of tolerance should occur only in the group that received a saline injection in the DE. Exposure to an occasion setter, without the CS, does not produce extinction (Rescorla, 1985, 1986). Therefore, the group that received exposure to the DE but did not receive a saline injection should not show extinction. If, however, the DE is the CS, as is widely assumed, then exposure to the DE, with or without an injection, should produce extinction.

\section{Method}

Subjects, apparatus, analgesia assessment, and drugs. The subjects were 38 male Sprague-Dawley rats weighing 240-260 g at the start of the experiment. They were housed as in Experiment 1. The apparatus, tail-flick procedure, and drug injections were the same as in Experiment 1.

Procedure. The rats were handled and weighed for 10 days prior to the experiment. They were then given three saline injections spaced $48 \mathrm{~h}$ apart. The rats were then randomly assigned to one of five groups for the training phase. Four groups received paired training $(n=8)$, and one group received unpaired training $(n=6)$. Because the purpose of this study was to examine changes in the paired groups as a function of the type of extinction training, the unpaired group was used as the control. There were eight training sessions, which were identical to those of Experiment 1. Forty-eight hours after the eighth acquisition session, the extinction sessions began. Since these procedures were found to be sufficient to demonstrate conditioned tolerance in Experiment 1, we did not test for conditioned tolerance after the acquisition phase. Because extinction did not appear to be complete in Experiment 1 (see Figure 2), we also decided to extend the extinction phase from four to nine sessions spaced 48 and $72 \mathrm{~h}$ apart. Two of the four groups that had received paired training remained in the colony room during the extinction phase. The rest (R) group was removed from the cage and handled. The rest injection (RI) group was removed from the cage and injected with saline. The other two paired groups received extinction sessions in the DE. The extinction (E) group was placed in its assigned box with no injection. The extinction injection (EI) group was placed in its assigned box after a saline injection. The unpaired (Unp) group was removed from the $\mathrm{HC}$ and handled. Forty-eight hours following the ninth extinction session, the rats were taken to the DE, given nicotine, and then given two tail-flick tests at 5 and 10 min postinjection.

\section{Results and Discussion}

As in the first experiment, the scores collected in the 5- and 10-min tail-flick tests did not differ appreciably, so they were combined. The median scores are presented in Figure 3, which shows that the unpaired group and the two paired groups exposed to the DE during the extinction phase (E and EI) had longer latencies than did the two paired groups that were not exposed to the DE after training (Groups R and RI). As in Experiment 1, there was significant heterogeneity of variance so the scores were analyzed with nonparametric tests. A Kruskal-Wallace test indicated significant group differences $\left(\chi^{2}=12.55\right.$, $p<.05)$. Mann-Whitney $U$ tests showed that Groups EI, E, and Unp all had significantly longer latencies than did Group RI (all $U_{\mathrm{s}} \leq 12$, all $p \mathrm{~s}<.05$ ) and Group R (all $U_{\mathrm{s}} \leq 11$, all $p \mathrm{~s}<.05$ ). In addition, Groups EI, E, and Unp did not differ significantly (all $p \mathrm{~s}>.5$ ), nor did Groups $\mathrm{RI}$ and $\mathrm{R}(p>.6)$. Thus, the unpaired and the two paired 


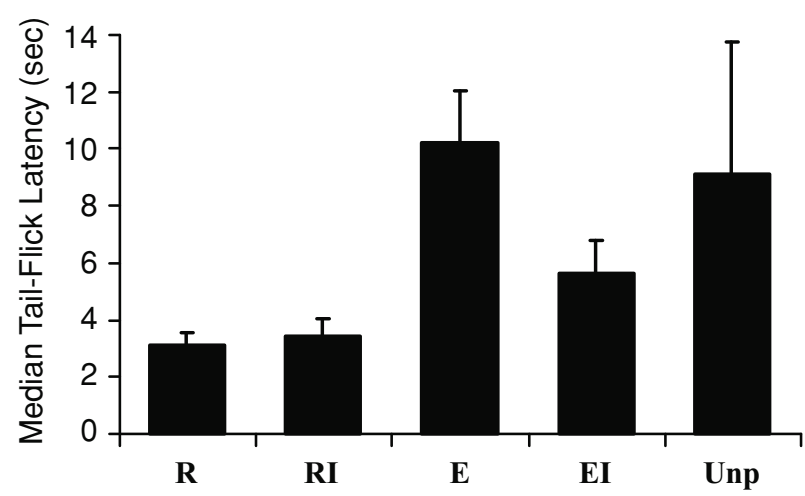

Figure 3. Median tail-flick latency in the postextinction test in Experiment 2. R, rest group; RI, rest-injection group; E, extinction group; EI, extinction-injection group; Unp, unpaired group. Error bars represent the semi-interquartile range.

groups (EI and E) that had extinction training in the DE were less tolerant to the analgesic effect of nicotine than were the two paired groups ( $\mathrm{RI}$ and $\mathrm{R}$ ) that did not receive extinction sessions in the DE. These results show that the DE served as a CS and not as an occasion setter. If the DE served as an occasion setter, then tolerance in Group E should not have been extinguished, which it clearly was. In addition, the lack of a significant difference between Groups EI and E and between Groups RI and R suggests that injection cues played no role in the development of conditioned tolerance. Finally, the low scores of Groups $\mathrm{R}$ and $\mathrm{RI}$ in comparison with Group Unp demonstrate that conditioned tolerance was retained during the 23 days of the extinction phase.

\section{GENERAL DISCUSSION}

The present experiments demonstrated the acquisition, retention, and extinction of conditioned tolerance to the analgesic effect of nicotine. Their findings are consistent with the previous research on this topic. The present study is the first to demonstrate extinction of conditioned tolerance to any effect of nicotine, as well as to demonstrate that conditioned tolerance is retained for over 3 weeks. These results suggest that, unless an extinction procedure is performed, tolerance will persist. The finding that conditioned nicotine tolerance can be extinguished is also consistent with previous research that has found extinction of conditioned tolerance to other drugs.

An interesting difference between the present experiments was seen in the tail-flick scores of the unpaired groups. In Experiment 1, they were very low, and we speculated that this might have been due to a form of conditioned tolerance in which early drug effects elicit compensatory responses that reduce the later, larger drug effects. However, there was less tolerance in the unpaired group in Experiment 2. An important difference was that in Experiment 1, there were 10 days of nicotine abstinence before the test, whereas in Experiment 2, there were 23 days. Perhaps after 10 days some nonassociative (functional or pharmacodynamic) tolerance persisted but was gone after 23 days (see Tiffany, Drobes, \& CepedaBenito, 1992, for a similar finding with morphine). As we discussed previously, Cepeda-Benito and Tiffany (1995) found that following unpaired training, a group that rested during a 30-day interval retained morphine tolerance. Another unpaired group, given saline injections during this period, lost tolerance. They attributed this to conditioning of the injection cues during the morphine training sessions. However, in the present studies, the unpaired group in Experiment 1 showed more tolerance than did the unpaired group in Experiment 2, even though the group in Experiment 1 received saline injections during the 10-day abstinence interval and the unpaired group in Experiment 2 did not. It is unlikely, therefore, that injection cues played any role in the unpaired groups in either study. Although it is risky to compare results across our two experiments, the most parsimonious explanation of the difference between the unpaired groups consists in the difference in abstinence intervals. The longer interval in Experiment 2 resulted in a loss of some type of nonassociative tolerance, either functional or dispositional.

The results of Experiment 2 support the notion that in the paired/unpaired design that is often used in studies of conditioned tolerance, the contextual cues serve as the CS. We found no evidence that the DE served as an occasion setter. In addition, the lack of contribution of injection cues is also consistent with conventional wisdom about conditioned tolerance studies (but see Cepeda-Benito \& Tiffany, 1995, for evidence that injection cues can condition tolerance in unpaired groups). That is, in the paired group in a drug tolerance study, saline injections prior to drug sessions and saline injections in the $\mathrm{HC}$ render the injection cues uninformative about the US as opposed to the DE and thus do not become conditioned (e.g., Kamin, 1969). The results of Experiment 2 also corroborate those of Deffner-Rappold, Azorlosa, and Baker (1996), who found, using a similar set of procedures, that conditioned morphine withdrawal was controlled by the DE, which functioned as a CS, not as an occasion setter. Also, as in the present Experiment 2, injection cues played no role.

However, this conclusion may only be true for studies in which the injection is given shortly after the entry into the context. In the present study, the rats were removed from the carrying case within 5-30 sec after arrival into the DE, injected, and immediately placed in the assigned box. A survey of the literature suggests that this procedure is common. However, some investigators deliberately insert a delay between arrival in the context and the injection (e.g., Falls \& Kelsey, 1989; Krank, 1987). It has been found that occasion setting is more likely to occur if there is a delay between the onset of the occasion setter and the onset of the CS (Holland, 1986). We are currently investigating whether inserting a delay between placement in the box and the injection will produce occasion setting by the DE. Because extinction of drug-related cues is an important component of drug abuse treatment (Siegel \& Ramos, 2002), an understanding of how contextual stimuli and other stimuli interact is crucial to the design of extinction 
therapies that also reduce the likelihood of relapse (Bouton \& Swartzentruber, 1991).

\section{REFERENCES}

BAKer, T. B., \& Tiffany, S. T. (1985). Morphine tolerance as habituation. Psychological Review, 92, 78-108.

Bevins, R. A., BesheER, J., \& PicKetT, K. S. (2001). Nicotine-conditioned locomotor activity in rats: Dopaminergic and GABAergic influences on conditioned expression. Pharmacology Biochemistry \& Behavior, 68, 135-145.

Bevins, R. A., \& Palmatier, M. I. (2003). Nicotine-conditioned locomotor sensitization in rats: Assessment of the US-preexposure effect. Behavioural Brain Research, 143, 65-74.

Bouton, M. E., \& Swartzentruber, D. (1986). Analysis of the associative and occasion-setting properties of contexts participating in a Pavlovian discrimination. Journal of Experimental Psychology: Animal Behavior Processes, 12, 333-350.

Bouton, M. E., \& Swartzentruber, D. (1991). Sources of relapse after extinction in Pavlovian and instrumental learning. Clinical Psychology Review, 11, 123-140.

Brooks, D. C., Karamanlian, B. R., \& Foster, V. L. (2001). Extinction and spontaneous recovery of ataxic tolerance to ethanol in rats. Psychopharmacology, 153, 491-496.

Caggiula, A. R., Donny, E. C., Chaudhri, N., Perkins, K. A., EvansMARTIN, F. F., \& SvED, A. F. (2002). Importance of nonpharmacological factors in nicotine self-administration. Physiology \& Behavior, 77, 683-687.

Caggiula, A. R., Donny, E. C., White, A. R., Chaudhri, N., Booth, S., GHARIB, M. A., ET AL. (2001). Cue dependency of nicotine self-administration and smoking. Pharmacology Biochemistry \& Behavior, 70, 515-530.

Caggiula, A. R., Donny, E. C., White, A. R., Chaudhri, N., Booth, S., Gharib, M. A., ET AL. (2002). Environmental stimuli promote the acquisition of nicotine self-administration in rats. Psychopharmacology, 163, 230-237.

Caggiula, A. R., Epstein, L. H., Antelman, S. M., Saylor, S. S., Knopf, S., Perkins, K. A., \& Stiller, R. (1993). Acute stress or corticosterone administration reduces responsiveness to nicotine: Implications for a mechanism of conditioned tolerance. Psychopharmacology, 111, 499-507.

Caggiula, A. R., Epstein, L. H., Antelman, S. M., Saylor, S. S., Perkins, K. A., KNopf, S., \& Stiller, R. (1991). Conditioned tolerance to the anorectic effect and corticosterone-elevating effects of nicotine. Pharmacology Biochemistry \& Behavior, 40, 53-59.

Caggiula, A. R., Epstein, L. H., \& Stiller, R. L. (1989). Changing environmental cues reduces tolerance to nicotine-induced anorexia Psychopharmacology, 99, 389-392.

Cepeda-Benito, A., Davis, K. W., Reynoso, J. T., \& Harraid, J. H. (2005). Associative and behavioral tolerance to the analgesic effects of nicotine in rats: Tail-flick and paw-lick assays. Psychopharmacology, 180, 224-233.

Cepeda-Benito, A., Reynoso, J. [T.], \& Erath, S. A. (2000). Doseresponse analyses of associative tolerance to nicotine analgesia in the rat: Tail-flick and hot-plate tests. Experimental \& Clinical Psychopharmacology, $\mathbf{8}, 112-116$.

Cepeda-Benito, A., Reynoso, J. [T.], \& McDaniel, E. H. (1998). Associative tolerance to nicotine analgesia in the rat: Tail-flick and hot-plate tests. Experimental \& Clinical Psychopharmacology, 6, 248-254.

Cepeda-Benito, A., \& Tiffany, S. T. (1995). Role of drug-administration cues in the associative control of morphine tolerance in the rat. Psychopharmacology, 122, 312-316.

Dafters, R., Hetherington, M., \& McCartney, H. (1983). Blocking and sensory preconditioning effects in morphine analgesic tolerance: Support for a Pavlovian conditioning model of drug tolerance. Quarterly Journal of Experimental Psychology, 35B, 1-11.

Davis, K. W., Cepeda-Benito, A., Harraid, J. H., \& Wellman, P. J. (2005). Plasma corticosterone in the rat in response to nicotine and saline injections in a context previously paired or unpaired with nicotine. Psychopharmacology, 180, 466-472.

Deffner-Rappold, C., Azorlosa, J. L., \& Baker, J. D. (1996). Ac- quisition and extinction of context-specific morphine withdrawal. Psychobiology, 24, 219-226.

Epstein, L. H., Caggiula, A. R., Perkins, K. A., McKenzie, S. J., \& Sмiтh, J. A. (1991). Conditioned tolerance to the heart rate effects of smoking. Pharmacology Biochemistry \& Behavior, 39, 15-19.

Epstein, L. H., Caggiula, A. R., \& Stiller, R. L. (1989). Environmentspecific tolerance to nicotine. Psychopharmacology, 97, 235-237.

Falls, W. A., \& Kelsey, J. E. (1989). Procedures that produce contextspecific tolerance to morphine in rats also produce context-specific withdrawal. Behavioral Neuroscience, 103, 842-849.

Fanselow, M. S., \& German, C. (1982). Explicitly unpaired delivery of morphine and the test situation: Extinction and retardation of tolerance to the suppressing effects of morphine on locomotor activity. Behavioral \& Neural Biology, 35, 231-241.

Grahame, N. J., Hallam, S. C., Geier, L., \& Miller, R. R. (1990). Context as an occasion setter following either CS acquisition and extinction or CS acquisition alone. Learning \& Motivation, 21, 237-265.

Greeley, J., \& Cappell, H. (1985). Associative control of tolerance to the sedative and hypothermic effects of chlordiazepoxide. Psychopharmacology, 86, 487-493.

GRIFFITHS, J. W., \& Goudie, A. J. (1986). Analysis of the role of drugpredictive environmental stimuli in tolerance to the hypothermic effects of the benzodiazepine midazolam. Psychopharmacology, 90, 513-521.

Grisel, J. E., Wiertelak, E. P., Watkins, L. R., \& Maier, S. F. (1994). Route of morphine administration modulates conditioned analgesic tolerance and hyperalgesia. Pharmacology Biochemistry \& Behavior, 49, 1029-1035.

Holland, P. C. (1983). Occasion setting in Pavlovian feature positive discriminations. In M. L. Commons, R. J. Herrnstein, \& A. R. Wagner (Eds.), Quantitative analyses of behavior: Discrimination processes (Vol. 4, pp. 183-206). New York: Ballinger.

Holland, P. C. (1986). Temporal determinants of occasion setting in feature-positive discriminations. Animal Learning \& Behavior, 14, 111-120.

KAMIN, L. J. (1969). Predictability, surprise, attention, and conditioning. In B. A. Campbell \& R. M. Church (Eds.), Punishment and aversive behavior (pp. 279-296). New York: Appleton-Century-Crofts.

Kim, J. A., Siegel, S., \& Patenall, V. R. A. (1999). Drug-onset cues as signals: Intraadministration associations and tolerance. Journal of Experimental Psychology: Animal Behavior Processes, 25, 491-504.

King, D. A., Bouton, M. E., \& Musty, R. E. (1987). Associative control of tolerance to the sedative effect of a short-acting benzodiazepine. Behavioral Neuroscience, 101, 104-114.

KranK, M. D. (1987). Conditioned hyperalgesia depends on the pain sensitivity measure. Behavioral Neuroscience, 101, 854-857.

MACRAE, J. R., \& SiEgel, S. (1987). Extinction of tolerance to the analgesic effect of morphine: Intracerebroventricular administration and effects of stress. Behavioral Neuroscience, 101, 790-796.

Mansfield, J. G., \& Cunningham, C. L. (1980). Conditioning and extinction of tolerance to the hypothermic effect of ethanol in rats. Journal of Comparative \& Physiological Psychology, 94, 962-969.

Millin, P. M., \& Riccio, D. C. (2002). Spontaneous recovery of tolerance to the analgesic effect of morphine. Physiology \& Behavior, 75, 465-471.

Paletta, M. S., \& Wagner, A. R. (1986). Development of contextspecific tolerance to morphine: Support for a dual-process interpretation. Behavioral Neuroscience, 100, 611-623.

Poulos, C. X., \& CAPpell, H. (1991). Homeostatic theory of drug tolerance: A general model of physiological adaptation. Psychological Review, 98, 390-408.

Ramos, B. M. C., Siegel, S., \& Bueno, J. L. O. (2002). Occasion setting and drug tolerance. Integrative Physiological \& Behavioral Science, 37, 165-177.

RAMSAY, D. S., \& Woods, S. C. (1997). Biological consequences of drug administration: Implications for acute and chronic tolerance. Psychological Review, 104, 170-193.

RESCORLA, R. A. (1985). Conditioned inhibition and facilitation. In R. R. Miller \& N. E. Spears (Eds.), Information processing in animals: Conditioned inhibition (pp. 299-326). Hillsdale, NJ: Erlbaum.

RESCORLA, R. A. (1986). Extinction of facilitation. Journal of Experimental Psychology: Animal Behavior Processes, 12, 16-24. 
SIEGEL, S. (1975). Evidence from rats that morphine tolerance is a learned response. Journal of Comparative \& Physiological Psychology, 89, 498-506.

SiEgEL, S. (1977). Morphine tolerance acquisition as an associative process. Journal of Experimental Psychology: Animal Behavior Processes, 3, 1-13.

SIEGEL, S. (1978). Tolerance to the hyperthermic effect of morphine in the rat is a learned response. Journal of Comparative \& Physiological Psychology, 92, 1137-1149.

Siegel, S. (1987). Pavlovian conditioning and ethanol tolerance. In K. O. Lindros, R. Ylikahri, \& K. Kiianmaa (Eds.), Advances in biomedical alcohol research (pp. 25-36). Oxford: Pergamon.

Siegel, S., \& Allan, L. G. (1998). Learning and homeostasis: Drug addiction and the McCollough effect. Psychological Bulletin, 124, 230-239.

Siegel, S., Baptista, M. A. S., Kim, J. A., McDonald, R. V., \& WeiseKelly, L. (2000). Pavlovian psychopharmacology: The associative basis of tolerance. Experimental \& Clinical Psychopharmacology, 8, 276-293.

Siegel, S., Hinson, R. E., \& Krank, M. D. (1981). Morphine-induced attenuation of morphine tolerance. Science, 212, 1533-1534.

Siegel, S., \& Ramos, B. M. C. (2002). Applying laboratory research:
Drug anticipation and the treatment of drug addiction. Experimental \& Clinical Psychopharmacology, 10, 162-183.

Siegel, S., Sherman, J. E., \& Mitchell, D. (1980). Extinction of morphine analgesic tolerance. Learning \& Motivation, 11, 289-301.

SwARTZENTRUBER, D. (1991). Blocking between occasion setters and contextual stimuli. Journal of Experimental Psychology: Animal Behavior Processes, 17, 163-173.

Swartzentruber, D. (1995). Modulatory mechanisms in Pavlovian conditioning. Animal Learning \& Behavior, 23, 123-143.

Tiffany, S. T., Drobes, D. J., \& Cepeda-Benito, A. (1992). Contribution of associative and nonassociative processes to the development of morphine tolerance. Psychopharmacology, 109, 185-190.

U. S. Department of Health \& Human Services (1997). Preliminary results from the 1996 National Household Survey on Drug Abuse. Washington, DC: Substance Abuse \& Mental Health Administration.

WAlter, T. A., \& Riccio, D. C. (1983). Overshadowing effects in stimulus control of morphine analgesic tolerance. Behavioral Neuroscience, 97, 658-662.

(Manuscript received April 5, 2005; revision accepted for publication January 19, 2006.) 